\title{
Téoros
}

Revue de recherche en tourisme

\section{Cinéma et désert}

De Tataouine à Ouarzazate

\section{Lionel Lapompe-Paironne}

Volume 30, numéro 1, 2011

Ciné-tourisme

URI : https://id.erudit.org/iderudit/1012112ar

DOI : https://doi.org/10.7202/1012112ar

Aller au sommaire du numéro

\section{Éditeur(s)}

Université du Québec à Montréal

ISSN

0712-8657 (imprimé)

1923-2705 (numérique)

Découvrir la revue

\section{Citer cet article}

Lapompe-Paironne, L. (2011). Cinéma et désert : de Tataouine à Ouarzazate. Téoros, 30(1), 90-98. https://doi.org/10.7202/1012112ar

\section{Résumé de l'article}

Les paysages cinématographiques, réels ou imaginaires, peuvent constituer un élément déclencheur de la mobilité touristique, comme tout support visuel. Landes, collines et montagnes de la série Lord of the Rings (Le Seigneur des anneaux), mais aussi déserts de Lawrence of Arabia ou de Star Wars (La Guerre des étoiles), autant de paysages à la fois réels et imaginaires suscitent l'attrait touristique. Les environs de Ouarzazate (Maroc) et de Tataouine (Tunisie) on servi et servent encore de toile de fond à des productions diverses et variées. Pourtant, si le cadre paysager reste identique, les conséquences sur l'aménagement touristique et sur la fréquentation ne sont pas les mêmes d'un bout à l'autre du Sahara, de même que les motivations des touristes qui se rendent sur ces deux sites. Cette contribution, après avoir traité de la manière dont l'industrie cinématographique a exploité les paysages désertiques du Maroc et de la Tunisie, aborde la question de l'importance différenciée du ciné-tourisme dans les deux espaces considérés. Il s'agit de comprendre, notamment grâce à la théorie des « marqueurs » de MacCannell (1976, r. 1989a), comment le tourisme lié au cinéma s'est développé selon deux modalités différentes, en termes de fréquentation et d'impact spatial, d'un bout à l'autre du Maghreb.
Ce document est protégé par la loi sur le droit d'auteur. L’utilisation des services d’Érudit (y compris la reproduction) est assujettie à sa politique d'utilisation que vous pouvez consulter en ligne.

https://apropos.erudit.org/fr/usagers/politique-dutilisation/ 


\title{
Cinéma et désert \\ De Tataouine à Ouarzazate
}

\author{
Lionel LAPOMPE-PAIRONNE \\ Agrégé de géographie, docteur en géographie \\ Université de Nice-Sophia-Antipolis \\ lapompe63@hotmail.com
}

\begin{abstract}
RÉSUMÉ: Les paysages cinématographiques, réels ou imaginaires, peuvent constituer un élément déclencheur de la mobilité touristique, comme tout support visuel. Landes, collines et montagnes de la série Lord of the Rings (Le Seigneur des anneaux), mais aussi déserts de Lawrence of Arabia ou de Star Wars (La Guerre des étoiles), autant de paysages à la fois réels et imaginaires suscitent l'attrait touristique. Les environs de Ouarzazate (Maroc) et de Tataouine (Tunisie) ont servi et servent encore de toile de fond à des productions diverses et variées. Pourtant, si le cadre paysager reste identique, les conséquences sur l'aménagement touristique et sur la fréquentation ne sont pas les mêmes d'un bout à l'autre du Sahara, de même que les motivations des touristes qui se rendent sur ces deux sites. Cette contribution, après avoir traité de la manière dont l'industrie cinématographique a exploité les paysages désertiques du Maroc et de la Tunisie, aborde la question de l'importance différenciée du ciné-tourisme dans les deux espaces considérés. II s'agit de comprendre, notamment grâce à la théorie des «marqueurs» de MacCannell (1976, r. 1989a), comment le tourisme lié au cinéma s'est développé selon deux modalités différentes, en termes de fréquentation et d'impact spatial, d'un bout à l'autre du Maghreb.
\end{abstract}

Mots-clés : Ciné-tourisme, pratiques touristiques, désert, Maroc, Tunisie.

Le cinéma et son industrie ont généré et génèrent des flux touristiques spécifiques dont la mesure demeure difficile. Motivation de voyage parmi d'autres ou véritable pèlerinage de fanatique averti, le "ciné-tourisme » tire son essence d'une certaine forme de "topophilie» au sens de Tuan (1974). Ce «désir d'ailleurs » induit par le cinéma peut s'exercer, géographiquement parlant, à toutes les échelles : urbaine, par exemple Montmartre, dans Le fabuleux destin d'Amélie Poulain (Freytag, 2008 : 17), jusqu'à l'échelle nationale avec le cas de la Nouvelle-Zélande, lieu de tournage de Lord of the Rings (Carla et al., 2007).

Le paysage, au sens large, joue ici un rôle important : « acteur », lui aussi (François, 1999: 87), il contribue en amont fortement à l'attrait touristique, cet attrait étant ensuite renforcé par l'œuvre cinématographique. Parmi ces paysages, les grandes étendues, les confins et les espaces «vierges» ou à faible empreinte sont privilégiés. Ainsi, Cazes (2005 : 125) rappelait la «recherche prioritaire des destinations étrangères, des confins et des extrêmes, du type des «déserts»». Les paysages désertiques, comme les espaces de montagne ou les forêts, font partie de ces décors susceptibles d'être rêvés puis investis physiquement par le biais du cinéma, notamment. Le désert est en effet présent dans un certain nombre de films, comme dans Land of the Pharaohs, de H. Hawks (1955), où «les scènes d'extérieur ne donnent que l'image de l'étendue du désert»(Liandrat-Guigues, cité dans Mottet, 1998 : 83), mais il a également servi de décor pour le tournage de films célèbres, tels que Lawrence of Arabia (1962) ou encore et surtout la série Star Wars (1977-2005).

Ce sont les paysages désertiques d'Afrique du Nord qui ont fait office de toile de fond paysagère à ces productions cinématographiques. Il s'agit plus précisément des environs de Ouarzazate, dans le Sud marocain, et de ceux de Tataouine, à quelques 500 kilomètres au sud de Tunis. Les lieux de tournage, bien que différents d'un lieu à l'autre, peuvent être visités et participent à l'activité touristique à leur manière au sein de ces deux États bien insérés dans la mondialisation touristique et particulièrement proches du foyer émetteur européen. Il s'agit donc de deux cas de ciné-tourisme induits par une ressource paysagère identique, à savoir le désert, au sens large. 


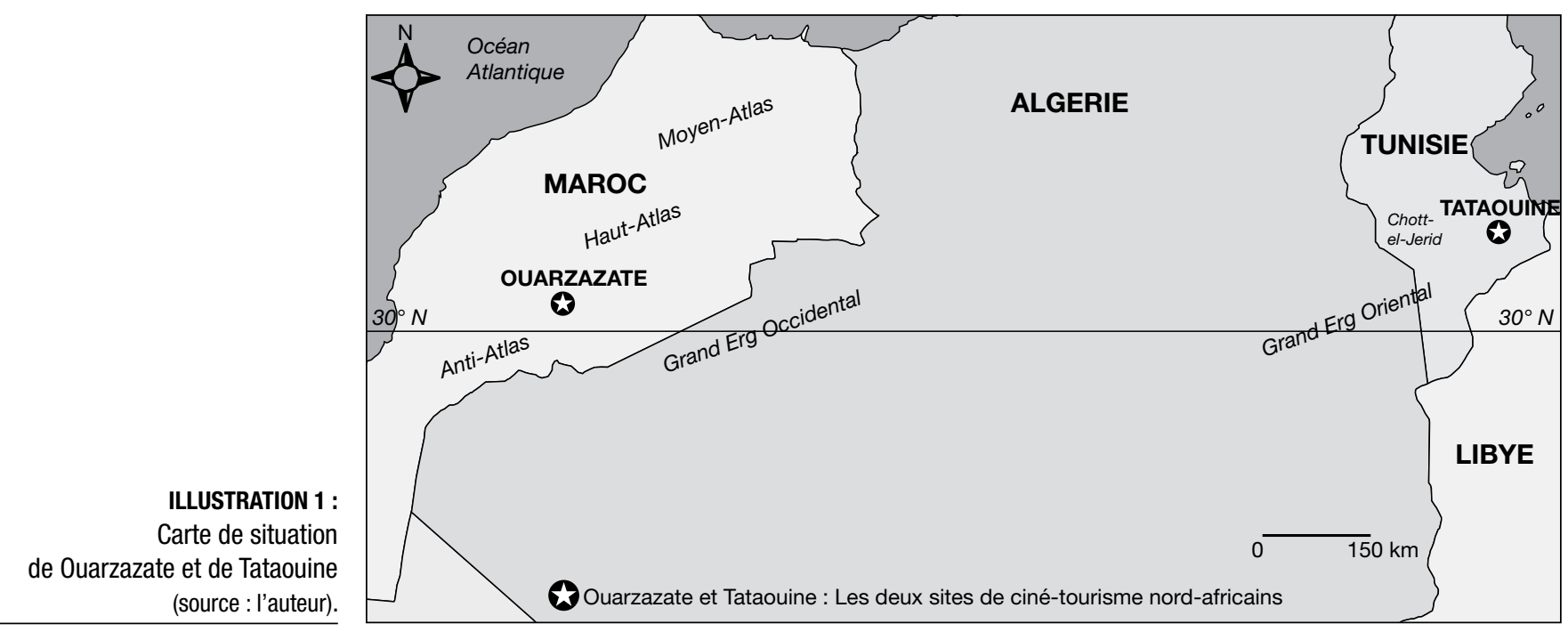

Il faut toutefois préciser le sens donné ici à l'expression "ciné-tourisme», terme encore peu utilisé dans les travaux en français. En effet, le passage du cinéma au tourisme peut se faire suivant deux modalités. En premier lieu, les évènements de type «festival» sont générateurs de flux touristiques : on pensera ainsi au cas d'Avoriaz (Desaubes, 1997) ou à Cannes. En second lieu, il y a la visite de lieux de tournage, voire de structures permanentes résultant des nécessités de tournage (Orueta et Valdés, 2007 : 185), comme c'est le cas à Hollywood, à Los Angeles, ou encore lorsqu'il s'agit de visiter les studios de Bombay. Nous entendons ici le ciné-tourisme au sens anglo-saxon de «movie-induced tourism» ou «filminduced tourism", à savoir la deuxième modalité (Riley et Van Doren, 1992; Riley et al., 1998; Busby et Klug, 2001).

C'est dans cette perspective théorique que cet article tente de cerner comment et dans quelle mesure l'exploitation cinématographique de certains paysages désertiques nord-africains a généré une fréquentation touristique et un aménagement différenciés. En effet, nous sommes en présence de deux cas semblables et différents, car si le désert a été exploité en tant que support paysager, il a servi de toile de fond à des paysages considérés comme "réels» d'un côté, et comme «imaginaires» de l'autre. Quelles sont les conséquences de cette différence d'exploitation sur la fréquentation touristique?

Pour répondre à cette question, on rappellera d'abord comment l'industrie cinématographique a "exploité» le désert dans les deux cas; puis on comparera le développement du ciné-tourisme sur les deux sites, en utilisant notamment la théorie des «marqueurs» touristiques (MacCannell, 1989a).

\section{Des paysages désertiques aux paysages cinématographiques \\ Un cadre naturel et paysager recherché par l'industrie cinématographique}

C'est aux environs de Ouarzazate et de Tataouine, toutes deux peuplées d'environ 60000 habitants, que se sont déroulés les tournages les plus significatifs dans le nord de l'Afrique (voir illustration 1).
Situées toutes deux au sud de deux anciennes colonies françaises, à des latitudes relativement similaires (30 degrés nord pour la première, 32 degrés nord pour la seconde), elles ont également comme point commun leur relative importance administrative, respectivement au sein de la province de Ouarzazate et du gouvernorat (ou wilaya) de Tataouine.

L'importance de ces deux sites pose la question de leurs points communs en termes paysagers au sens large : quels facteurs peuvent expliquer leur élection pour divers tournages?

Les environs des deux localités sont caractérisés par des paysages façonnés par l'aridité. D'un côté on trouve le Sud tunisien, aux confins de la Libye à l'ouest, et de l'Algérie à l'est, qui est caractérisé par un climat aride aux alentours de Tataouine. Nous sommes ici à l'est de la région la plus méridionale de Tunisie, au sud du Chott-el-Jérid et en bordure du Grand Erg oriental. Du côté marocain, Ouarzazate se situe dans une province englobée dans la vaste région de Souss-Massa-Drâa, allant de l'Atlantique (Agadir) à la frontière algérienne (province de Zagora). Il s'agit donc de deux «portes du désert» au sens large, comme les promoteurs touristiques en tous genres aiment le clamer (Bouaouinate, 2008 : 54). Le climat y est assez aride pour évoquer le «vrai» désert, mais elles ne sont pas aussi isolées que des oasis à proprement parler comme Douz, Ksar Ghilane ou Zagora, où l'influence du désert se fait beaucoup plus sentir. Pas vraiment situés "en plein désert ", mais bénéficiant de sa proximité, les deux localités et leurs environs ont rendu et rendent bien des services à l'industrie cinématographique comme à l'industrie touristique.

Au-delà de l'aspect physique, la composante architecturale est également très importante dans le choix de ces lieux ciné-touristiques. Les ksour et casbahs sont en effet des éléments culturels et architecturaux typiques mis en valeur par le cinéma et le tourisme. Ainsi, les ksour, villages fortifiés comprenant généralement des greniers et des habitations, sont une des attractions touristiques les plus emblématiques du Sud tunisien, où on en dénombre 150 environ, principalement entre Matmata et Tataouine. Dans les environs 
de Ouarzazate, ce sont les casbahs qui font l'objet de cet engouement, bien qu'on dénombre également un nombre important de ksour, mais en quantité nettement moins importante qu'au sud de la Tunisie. La ville est en effet située sur la Route des mille casbahs, en réalité un ensemble de plusieurs routes situées à l'intérieur d'un triangle à peu près délimité par Marakkech, Errachidia et Mhamid (Popp, cité dans Minvielle et al., 2007 : 583).

Enfin, au-delà des paysages désertiques au sens large (c'est-à-dire le désert «physique», mais aussi «culturel»), Tataouine comme Ouarzazate bénéficient d'une bonne accessibilité routière, élément indispensable pour envisager un tournage, de même qu'une situation géopolitique relativement favorable par rapport aux voisins mauritanien, libyen et algérien, notamment depuis le début des années 1990 pour ce dernier.

\section{L'utilisation du paysage désertique comme décor cinématographique}

Des tournages plus ou moins importants ont donc eu lieu récemment au cœur de ces deux sites considérés comme des portes d'entrée vers le monde du désert. À Ouarzazate, il s'agit d'une production filmique variée, comprenant des productions à grande audience mondiale tels que Gladiator, de Ridley Scott (2000), mais aussi des productions moins «hollywoodiennes" comme Astérix \& Obélix : Mission Cléopâtre (2002), ou encore Les Rois Mages (2001) et Il Tempo dell'amore (1999). En revanche, Tataouine est célèbre dans le monde cinématographique principalement pour avoir servi de décor au film Star Wars Episode IV : A New Hope (1997). Il y a donc une différence fondamentale entre les deux utilisations de la "ressource désert» car, si à Ouarzazate l'environnement paysager sert de décor «réel», il n'en est pas de même à Tataouine, qui a servi à la mise en scène d'un décor imaginaire.

Le cinéma, dans les deux cas, a rapidement été approprié comme un élément «local», faisant office d'attraction touristique. Ceci n'est pas surprenant si on considère que la ressource paysagère originale est la motivation principale de l'industrie cinématographique. Ainsi, cette ressource, déjà mobilisée par les acteurs locaux et internationaux de la promotion touristique, acquiert par le biais du cinéma une dimension supplémentaire dans l'image dont elle bénéficie auprès des touristes. Visiter le désert, c'est bien, mais visiter les lieux de tournage de tel ou tel film dans le désert, c'est encore mieux. Ainsi, Bleasdale (2006: 450), à propos du cas tunisien, insiste sur cette appropriation rapide : «[T]hese locations have been very quickly adopted as part of Tunisia's heritage and incorporated into the tourist provision." Le cinéma fait donc partie du décor touristique, et les sites Internet de promotion générale et touristique des deux localités (tataouine.org et ouarzazate.com) jouent une partie de leur stratégie de "marketing urbain» sur cette ressource. Ainsi, on proclame fièrement que Ouarzazate est une "cité de cinéma», et que "les studios Atlas peuvent être visités quand il n'y a pas de tournage en payant la somme de $30 \mathrm{DH}$, mais [que] ses décors valent vraiment le détour ». De même, on trouve sur le même site Web la liste des films tournés aux alentours de la ville entre 1984 et 2004. En revanche, le site dédié à Tataouine ne présente pas de promotion particulière de Ksar Hadada, principal site de tournage local. En effet, le décor de Star Wars est en quelque sorte «noyé» dans la multitude de sites fortifiés à visiter dans les environs, ce qui en fait un site moins emblématique que les studios de production marocains.

\section{Un inégal développement de l'activité cinématographique d'un bout à l'autre du Maghreb} L'activité touristique générée par la sphère cinématographique s'est très inégalement développée entre Tataouine et Ouarzazate. Dans un des cas (Ouarzazate), le cinéma a en effet entraîné des aménagements permanents, tandis qu'à l'autre extrémité du Maghreb, seul le décor est resté.

À Ouarzazate, une véritable infrastructure «lourde» (en comparaison avec son homologue tunisienne) existe aujourd'hui. Il s'agit en effet de studios, dont le principal, situé à la sortie de la ville, se nomme Atlas Corporation Studios. Étendu sur une cinquantaine d'hectares, il a été érigé en 1983 à l'initiative de Mohamed Belghmi (Goldsmith et O'Regan, 2003 : 107). Disparu en 2003, ce dernier était considéré comme "l'un des pionniers de l'hôtellerie et du tourisme marocains" (Anonyme, $2003: 39$ ). Ainsi, dès le début des années 1980, la naissance de l'infrastructure cinématographique est liée, par le biais des acteurs locaux, à l'industrie touristique. L'objectif est de proposer aux réalisateurs internationaux l'utilisation d'installations permanentes sur place (studios, mais aussi infrastructures d'hébergement). La création des Studios Atlas intervient à la suite des premiers tournages effectués dans la région dans les années 1960. Ainsi le film Lawrence of Arabia, de David Lean (1962), avait déjà bénéficié des décors des alentours de Ouarzazate, notamment la casbah d'Aït Benhaddou ou encore celle de Tifoultoute (environ huit kilomètres à l'ouest de Ouarzazate). Les Studios Atlas ont fait par la suite tache d'huile, puisque d'autres studios ont été créés sur place. C'est le cas des Studios Kanzaman, hébergeant un centre de formation aux métiers du cinéma, et des Studios CLA. Cependant, ce sont les Studios Atlas qui demeurent à l'heure actuelle la seule véritable attraction touristique parmi ces structures locales, même si les autres peuvent également être visités. La dynamique du développement touristique lié au cinéma doit également beaucoup à la présence à Ouarzazate d'un aéroport international, où opère entre autres la Royal Air Maroc, vers les principales destination européennes (Londres, Paris, Bruxelles, Amsterdam) et nationales (Agadir, Casablanca).

Le développement de l'activité cinématographique n'est pas aussi ancien et important à Tataouine. Pour cette dernière, contrairement à Ouarzazate, ce ne sont pas des studios et un ensemble de films divers, mais un film, ou plus précisément une saga cinématographique, qui est au cœur du ciné-tourisme. Tataouine et ses environs ont en effet servi à représenter le décor de la planète Tatouïne dans la saga Star Wars, portée à l'écran par G. Lucas. Tournée en six épisodes entre 1977 et 2005, la suite peut être présentée comme une double trilogie, les épisodes IV, V, et VI étant 
sortis les premiers, suivis de la trilogie précédente, c'est-àdire les épisodes I, II et III. Le Sud tunisien est popularisé par Georges Lucas dès 1976, pour représenter la fameuse planète dans Star Wars, Episode IV : A New Hope, sorti sur les écrans en 1977. Planète dont est originaire la famille Skywalker, Tatouïne évoque bien sûr la ville dont elle est le presque homonyme. Dans la longue histoire de Star Wars, il s'agit d'une planète désertique, très difficilement vivable, et servant de refuge pour toutes sortes d'individus infréquentables. Éclairée par deux soleils, la planète possède sa propre langue, dont les dialogues sont parfois doublés lorsque nécessaire (Pierozak et De Robillard, 2004 : 335). La planète, présente dans la quasi-totalité des épisodes de la saga (cinq sur six), est donc apparentée à un désert de science-fiction, où les étendues arides, les dunes de sable et les canyons servent de toile de fond à des journées très chaudes et des nuits très froides. Très aride, on peut survivre en récupérant de la vapeur d'eau en plus ou moins grande quantité, comme le fait le personnage Owen Lars au sein d'une exploitation conséquente.

Les paysages de la planète Tatouïne sont ceux du Sud tunisien, plus précisément autour de Tataouine (la ville et ses environs), mais aussi jusqu'à Matmata et Tozeur, où les paysages désertiques sont parfaitement conformes à ceux imaginés pour la planète en question, même si certaines scènes se déroulant sur Tatouïne ont été tournées non pas en Tunisie, mais dans l'Arizona (Return of the Jedi, 1983).

Les paysages ne sont pas l'unique motivation qui explique le choix du réalisateur. En effet, l'habitat traditionnel des ksour a été utilisé, principalement celui de Ksar Hadada, qui apparaît à peu de choses près sans retouches à l'écran (voir illustration 2). On aperçoit au premier plan du cliché les habitations du ksar tel que l'on peut les visiter aujourd'hui, laissées quasiment en l'état après le tournage. À l'écran, c'est surtout la fameuse «ferme d'humidité», où la famille Lars «cultive» littéralement de l'humidité, qui a été mise en scène grâce aux installations du ksar telles que vues sur la photographie. Sur cette planète Tatouïne, désertique au sens propre du terme, cette exploitation est souterraine, et sert à la fois de lieu de production de l'humidité et de lieu d'habitation pour l'ensemble de la famille. L'intérieur à ciel ouvert de l'ancien grenier fortifié a servi à représenter la cour centrale, à l'air libre, de cette gigantesque habitation souterraine, tandis que les portes d'entrées des différentes chambres du ksar ont été utilisées pour jouer le rôle des entrées des tunnels conduisant à l'intérieur même des différentes pièces.

Les villes de Ouarzazate et de Tataouine sont donc deux cas intéressants, en ce sens où elles constituent à la fois des points de rayonnement touristique et des lieux centraux de tournages de films. Situées toutes deux aux portes du désert et à proximité immédiate de lieux fortement récepteurs du tourisme international (Djerba, Agadir), toutes deux n'ont pas connu un développement identique des infrastructures locales d'hébergement. Cependant, elles ont en commun une fréquentation touristique en lien avec l'activité cinématographique. Comment se manifeste cet impact du cinéma sur l'activité touristique proprement dite, et comment celle-ci se manifeste-t-elle dans les paysages?

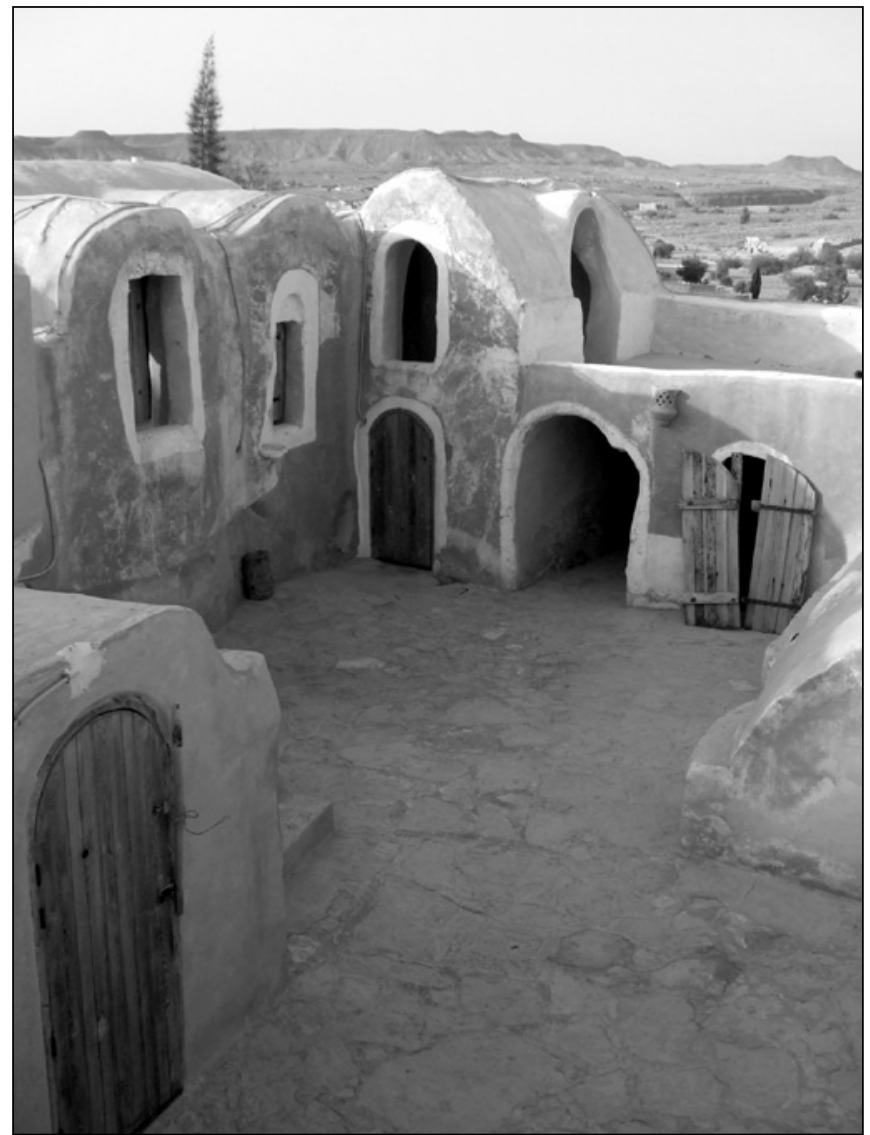

ILLUSTRATION 2 : Un aperçu de Ksar Hadada, utilisé comme décor de la planète Tatouïne dans la saga Star Wars (photo : Lionel Lapompe-Paironne).

Des paysages réels, des paysages imaginaires : des paysages cinématographiques à l'activité touristique

Le premier problème consiste à mesurer la réelle «motivation cinématographique» des touristes : peut-on dire que le décor de film est une motivation de voyage en tant que telle, ou une simple attraction parmi d'autres au niveau local ou national? Cette difficulté de la «mesure de l'impact» est bien montrée par Busby et Klug (2001 : 323), qui prennent comme exemple le cas du film britannique Notting Hill (1999), de Roger Michell. Leur étude montre que la visite de Kenwood House, un des lieux de tournage du film, a connu une hausse de fréquentation de $10 \%$ en août 1999. En revanche, elle montre aussi que si $88,7 \%$ des touristes interrogés ont vu l'affiche du film et que $55 \%$ d'entre eux ont vu le film. Seuls $5 \%$ reconnaissent s'être déplacés spécifiquement après avoir vu le film. De plus, l'étude est circonscrite à ce seul lieu alors que le film a été tourné en divers endroits de Londres. Il est donc difficile d'enquêter sur l'ensemble des touristes se rendant à Londres, et seuls quelques sites emblématiques comme Kenwood House peuvent servir de base d'analyse. Le problème de la mesure de l'impact se pose donc autant ici que dans le cas de Notting Hill : tout comme dans l'étude 
de Busby et Klug, on peut penser que le ciné-tourisme est englobé dans d'autres pratiques touristiques et dans d'autres échelles géographiques plus larges (celles de la ville, de la région et du pays).

On peut mesurer l'impact du cinéma sur l'activité touristique grâce à trois indicateurs : la présence plus ou moins importante d'hébergements à proximité des sites; la présence d'excursions/activités spécifiquement liées au cinéma chez les voyagistes; enfin, la présence du cinéma dans ce que D. MacCannell (1976, r. 1989a) nomme les «marqueurs» touristiques, et qui seront ici subdivisés, comme le suggère ce dernier, en marqueurs on-sight et off-sight.

\section{Les structures d'hébergement et le ciné-tourisme à Tataouine et Ouarzazate}

La présence d'hébergements spécifiquement reliés au cinétourisme peut constituer la première manière de mesurer cet impact. En Tunisie, Ksar Hadada, l'ancien village fortifié ayant servi à représenter les décors extérieurs de Tatouïne et représenté sur l'illustration 2, a été en partie transformé en hôtel, mais celui-ci a rapidement été fermé. Les touristes peuvent aujourd'hui séjourner à l'hôtel Sidi Driss, à Matmata, afin de revivre une partie de leur film favori. En effet, l'hôtel, surnommé Star Wars Hotel, est un habitat troglodytique traditionnel utilisé tel quel pour servir à représenter la propriété du personnage de Lars Owen dans Star Wars. L'intérieur a été rénové et repeint afin de coller au maximum avec les décors du film. On peut aussi y retrouver l'ensemble des pièces qui forment l'habitation de Lars Owen, jusqu'à la salle à manger. Cependant, il ne s'agit pas des lieux qui ont directement servi au tournage des scènes du film, mais d'une reconstitution.

Cette transformation supposée de décors filmiques en infrastructures d'hébergement ne se retrouve pas à Ouarzazate, où fleurissent de nombreux hôtels en tous genres, y compris de luxueux centres de villégiature, tels que le Riad Salam ou le Framissima Karam. Ici, plutôt que de rechercher la reconstitution de décors dans l'hébergement, il s'agit plutôt de proposer des séjours de luxe avec possibilité de visiter les studios. Les premiers tournages ont engendré le développement des studios qui, à leur tour, ont généré le développement de l'infrastructure touristique, puis l'activité touristique en elle-même. Contrairement à Tataouine, où les passionnés d'un film précis recherchent assidûment les éléments de décor, à Ouarzazate le cinéma apparaît plus comme une motivation secondaire. Cette différence s'explique par le fort impact de Star Wars, seul film emblématique du Sud tunisien, dans de nombreux pays occidentaux, tandis qu'aucun autre film tourné dans le Sud marocain n'a généré un tel engouement et un tel fandom international. Ainsi, Brooker (2002: 15) rappelle que, dans les pays occidentaux, même les profanes connaissent vaguement des éléments typiques du film, tels que Dark Vador, ou encore la célèbre formule "May the Force be with you». Il faut également ajouter, dans le cas du Sud marocain, l'importance du développement hôtelier lié non pas au cinétourisme, mais à l'activité cinématographique en tant que telle. On peut alors parler d'hébergement «d'affaires», en ce sens que l'infrastructure d'hébergement s'adresse aux «voyageurs» au sens large, mais aussi plus directement aux travailleurs de l'industrie du cinéma. Une grande partie de la communication (sites Internet des deux localités) autour de la ville de Ouarzazate, qu'elle soit le fait d'instances locales ou nationales, met en avant cette disponibilité immédiate de structures d'hébergement.

Pour autant, il n'existe pas ou plus de transformation directe de structures de tournage en structures d'hébergement, et les Studios Atlas comme les lieux de tournage de Star Wars demeurent des attractions qui ne font que se visiter, sans s'habiter temporairement.

\section{Le ciné-tourisme au Maghreb chez les voyagistes}

Le contenu des séjours et circuits proposés par les voyagistes à destination du Maghreb est une deuxième manière de tenter de mesurer l'importance du ciné-tourisme autour de Ouarzazate et de Tataouine. Ainsi, quelle est la place accordée au ciné-tourisme tunisien et marocain par l'industrie du voyage au sens large? Il s'agira donc ici de s'intéresser à un tourisme principalement international, au départ du foyer émetteur français. Ce choix est ici lié à des contraintes matérielles de recueil des données, et il s'agit d'avoir une idée générale de la place du ciné-tourisme dans le contenu d'un corpus de catalogues francophones. Les Français constituent en effet la principale clientèle touristique internationale du Maroc (Brault, $2004: 8$ ) et de la Tunisie.

La première constatation importante est qu'il n'existe pas en France, à notre connaissance, de voyagistes dans le ciné-tourisme, contrairement à d'autres pratiques touristiques spécifiques. Cette absence renforce l'idée que le cinétourisme serait une activité touristique parmi d'autres, mais pas une pratique touristique à proprement parler. Du moins, c'est une hypothèse, mais qui reste difficile à vérifier, et il y a fort à parier qu'un pourcentage plutôt restreint des touristes le fait uniquement à des fins de ciné-tourisme (Busby et Klug, $2001: 323$ ). Il est donc nécessaire de chercher chez les voyagistes classiques l'insertion, à l'intérieur de séjours plus généralistes, d'activités ressemblant de près ou de loin à du ciné-tourisme. Nous nous sommes donc penchés sur le contenu des séjours au Maroc et en Tunisie proposés par sept voyagistes français, chez qui nous avons procédé en deux étapes : recensement du nombre de séjours comprenant Ouarzazate ou Tataouine dans un premier temps; puis recherche d'éléments mentionnant le cinéma au sein de ces séjours dans un second temps. En termes quantitatifs, la différence principale entre les deux destinations réside dans le plus grand nombre de séjours pris en compte dans le cas marocain. Ceci s'explique par la combinaison séjours/ circuits (on peut insérer Ouarzazate dans un tour du Maroc, tout comme on peut y séjourner en centre de villégiature). En Tunisie, par contre, nous n'avons relevé que des voyages de type circuit (voir tableau 1).

On constate donc une faible mobilisation de la ressource "cinéma» dans les programmes de séjours ou de circuits de ces deux pays/destinations. Celle-ci est systématiquement évoquée, jamais vraiment proposée comme une activité à part entière, y compris pour des excursions de faible durée. C'est peut-être là un élément d'explication, le faible temps 
Tableau 1: Comparaison entre le nombre de séjours offerts avec ou sans activités liées au ciné-tourisme pour le marché français, au Maroc et en Tunisie

\begin{tabular}{l|c|c}
\hline & $\begin{array}{c}\text { Nombre de séjours/circuits } \\
\text { (sans ciné-tourisme) }\end{array}$ & $\begin{array}{c}\text { Nombre de circuits/séjours } \\
\text { (intégrant le ciné-tourisme) }\end{array}$ \\
\hline $\begin{array}{l}\text { Ouarzazate, } \\
\text { MAROC }\end{array}$ & $41(87,3 \%)$ & $6(12,7 \%)$ \\
\hline $\begin{array}{l}\text { Tataouine, } \\
\text { TUNISIE }\end{array}$ & $28(87,5 \%)$ & $4(12,5 \%)$ \\
\hline
\end{tabular}

Source : Look Voyages, Fram, Thomas Cook, Jet Tours,

Marmara, Kuoni, Nouvelles Frontières, 2009.

consacré aux visites liées au cinéma ne permettant pas de construire une véritable pratique touristique. Les voyagistes préfèrent alors miser sur des pratiques plus rentables en termes de durée et d'investissement. Ainsi, un tour des oasis du Sud tunisien sera favorisé au détriment d'une simple visite de Ksar Hadada. Cette dernière sera donc intégrée dans une excursion à l'échelle plus conséquente. Pourtant, le contenu des catalogues des voyagistes révèle malgré tout une utilisation de la ressource cinéma à des fins purement paysagères. Les décors cinématographiques ne sont pas vantés en tant que tels (par exemple tel ou tel élément précis de tel film, dune, maison traditionnelle évoque tel ou tel personnage etc.), mais uniquement comme caution au paysage lui-même. En d'autres termes, ce n'est pas le paysage désertique de film qui est vanté, mais le désert lui-même, le cinéma ne venant que conforter la valeur du paysage. Ainsi peut-on lire dans le catalogue du voyagiste Kuoni à propos de Ouarzazate : "Scène ayant servi dans beaucoup de films d'aventures et de films ayant la nature pour thématique - Laurence d'Arabie, Gladiateur et la Guerre des Étoiles - les grandioses plaines du désert servent de décor impressionnant. Visitez les décors des films monumentaux d'Hollywood ayant été laissés sur place».

Chez les principaux voyagistes, en Tunisie comme au Maroc, les visites de sites ciné-touristiques s'effectuent au départ de Djerba, Agadir ou Ouarzazate. Le contenu descriptif des excursions au départ de ces sites ne comportent que très rarement des mentions relatives au cinéma. Ainsi, chez Fram, les excursions portent des intitulés généraux ne mentionnant pas spécifiquement l'intérêt cinématographique que peut avoir Ksar Hadada par exemple : «Tataouine, Chenini et première nuit sous des tentes berbères, aux portes du désert»; «Raid des oasis »; «traversée du Chott El Djerid et découverte des oasis de montagne» (extraits du catalogue numérique Fram). Chez Marmara, même si les descriptifs sont moins généralistes, la mention de Star Wars est absente :

Départ pour Tataouine et visite du marché aux épices.

Visite de Ghorfas et du musée Ksar Ouled Dabbab.

Découverte des villages berbères de montagne et du village semi-troglodytique de Chenini [...] Départ pour Gabès, puis Tozeur, en traversant le lac salé Chott el Djerid. Continuation pour Chebika et découverte des magnifiques oasis de montagne en $4 \times 4$. Arrivée à
Douz, «la porte du désert», puis à Matmata, le village aux allures lunaires avec ses habitations troglodytiques (extrait du catalogue numérique Marmara).

Ainsi, chez les professionnels du tourisme, le ciné-tourisme n'apparaît pas au Maroc et en Tunisie comme un élément essentiel du sight-seeing, ni comme une pratique touristique à part entière, mais plutôt comme un type de visite parmi d'autres, réservé à des initiés. Par exemple, si Ksar Hadada fait partie des ksour à visiter, le fait qu'il ait servi de décor à Star Wars n'est que très rarement mentionné. De même, Ouarzazate est juste mentionnée comme «décor naturel », comme «toile de fond », ayant servi pour des tournages de films, mais pas comme une attraction ciné-touristique en tant que telle.

\section{À la recherche de «marqueurs» touristiques sur place}

Les marqueurs, selon D. MacCannell (1976, r. 1989a : 110), sont l'ensemble des éléments d'un lieu qui, sur place ou non, servent d'intermédiaire pour informer le touriste. Partant du principe théorique que le premier contact entre le touriste et le lieu ne se fait jamais sur le lieu lui-même mais par l'intermédiaire de sa représentation, les marqueurs peuvent donc être à la fois sur le lieu lui-même (marqueurs on-sight) comme extérieurs à ce lieu (marqueurs off-sight).

MacCannell insiste particulièrement sur l'importance des marqueurs sur place, tels que les plaques commémoratives, les panneaux d'information ou de direction, qui prennent autant de sens que le lieu en lui-même. On les photographie autant que l'attraction touristique du lieu, et certains touristes vont même parfois jusqu'à les voler pour les emporter chez eux en guise de trophée commémoratif, ce qui montre leur signification. En les rapportant avec soi, on rapporte avec soi une partie du lieu visité (MacCannell, 1989a : 110).

Reprise par des auteurs français (Rieucau et Lageiste, 2006), l'analyse des «marqueurs» du paysage, ou plus globalement l' «empreinte» spatiale du tourisme, constitue une troisième façon d'avoir une idée de la manière dont le cinéma influe sur l'activité touristique. Il s'agit ici de repérer la présence ou l'absence d'éléments visibles contribuant à guider le touriste. Il peut exister des sentiers thématiques, des panneaux indicateurs, des routes à thème, autant de marques et d'empreintes visibles du ciné-tourisme.

Cette empreinte est relativement faible dans le Sud tunisien, et se limite à quelques panneaux à visée informative (voir illustration 3).

On peut voir sur cette illustration un panneau situé à l'entrée du ksar, marquant clairement le sens ciné-touristique du lieu. Pour autant, il demeure bien isolé, car la visite de l'intérieur de l'ancienne habitation fortifiée est dépourvue de tout autre panneau indicateur, mentionnant par exemple l'importance de tel ou tel bâtiment pour le tournage, ou de telle ou telle partie du ksar pour représenter un élément particulier à l'écran. Le visiteur en est donc réduit à imaginer par lui-même l'adéquation entre le lieu qu'il arpente et son utilisation dans un film. En tant que lieu, Ksar Hadada apparaît donc comme un "marqueur symbolique» au sens de la théorie de marqueurs de MacCannell (1989a : 111-112), car 


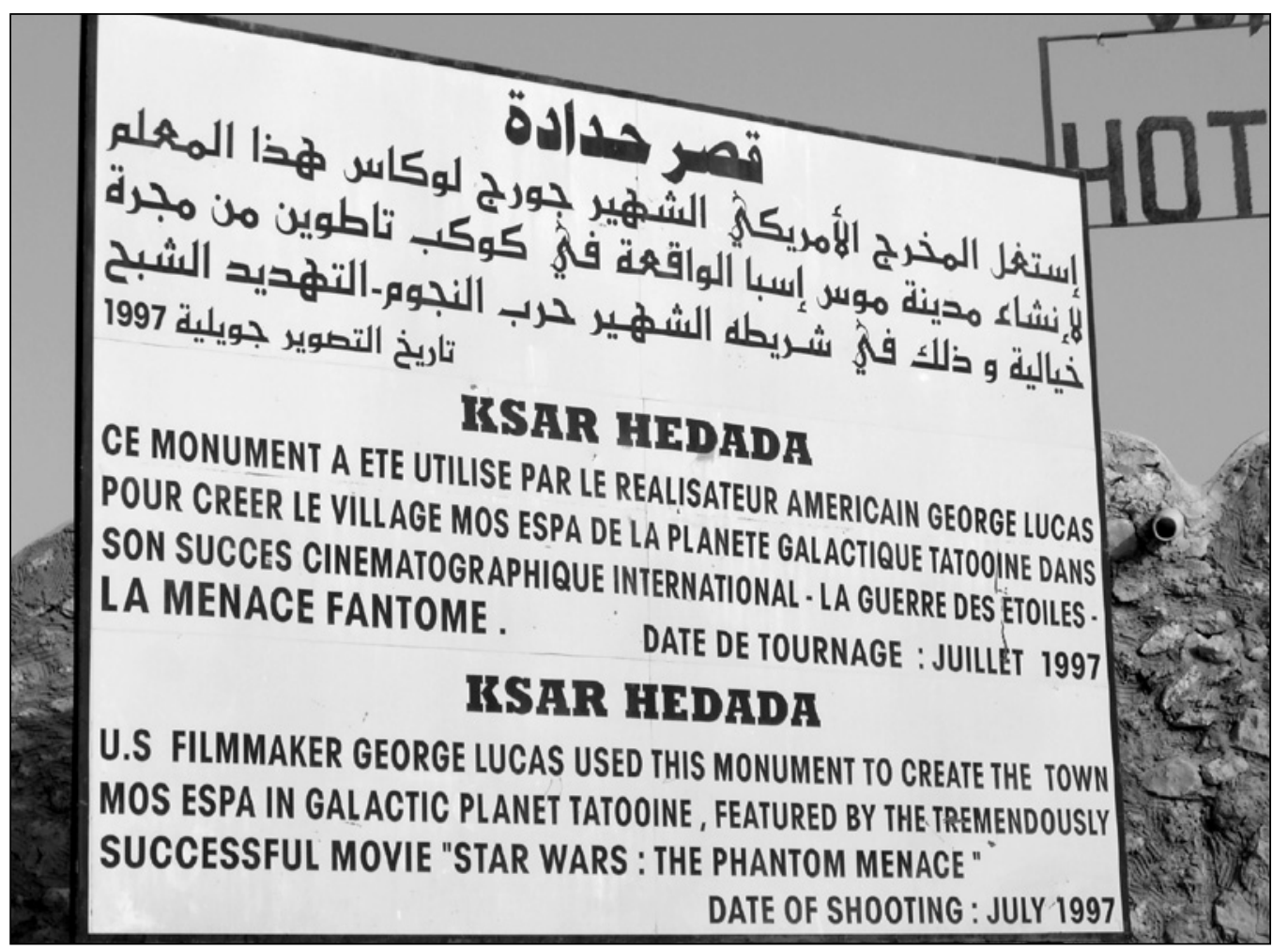

ILLUSTRATION 3 : Panneau à l'entrée de Ksar Hadada (photo : Lionel Lapompe-Paironne). il constitue un fragment d'un lieu qui donne sens à ce lieu tout entier : ainsi, en visitant Ksar Hadada, on visite le Sud tunisien, tout comme on visite San Francisco en se rendant, symboliquement, sur le Golden Gate Bridge, pour reprendre l'exemple de MacCannell. Il s'agit cependant d'un marqueur original, car autant un marqueur de l'espace « réel» (le désert du Sud tunisien) qu'un élément d'un espace imaginaire, la planète Tatouïne.

Dans le cas des Studios Atlas, on n'observe là aussi pas ou très peu de marqueurs sur place. Ici, pas de panneau solennel. Ce sont les guides locaux qui rappellent avec fierté que d'importantes équipes de tournage ont posé leurs valises ici. Seules les statues géantes de pharaons qui trônent à l'entrée des Studios Atlas rappellent que le visiteur entre dans un espace dédié à des décors factices. Temples égyptiens ayant servi de décors à The Mummy, palais de Cléopâtre reconstitué, décors moyenâgeux de Kingdom of Heaven, ou encore temples bouddhistes, ce ne sont pas, contrairement à Tataouine, des éléments culturels locaux qui ont été mis à contribution pour créer un paysage imaginaire, mais l'environnement lui-même. Le désert est ici recherché pour sa luminosité, la couleur du sol, les dunes ou rochers environnants, auquel on a surimposé des décors reconstitués. On ne s'étonne donc pas de ne pas relever de marques d'information sur un film symbolique comme Star Wars, puisque les Studios Atlas ne représentent pas un espace directement visualisé dans un film en tant que tel. Ainsi, si on peut véritablement s'imaginer être sur Tatouïne à Ksar Hadada, il n'en va pas de même aux Studios Atlas où les décors apparaissent à l'évidence montés de toutes pièces, au sens propre du terme.
Les marqueurs du ciné-tourisme dans les guides de voyage

Les marqueurs peuvent également être extérieurs, ou off-sight, comme le rappelle MacCannell (1989a : 110), pour qui «the use of the term extends it to cover any information about a sight, including that found in travel books and museum guides». La mention d'une attraction touristique dans les guides de voyage, en informant sur la "nécessité » de s'y rendre pour le touriste, participe pleinement à marquer un lieu ou un site, et s'inscrit pleinement dans la théorie des marqueurs touristiques.

On s'intéresse donc ici principalement (mais pas uniquement) aux touristes dits «individuels», ne voyageant pas systématiquement par l'intermédiaire d'un voyagiste, et plus particulièrement aux plus individuels d'entre eux. Selon la typologie de Cohen (1972), on parlerait au choix de individual mass tourists, d'explorers, ou encore de drifters suivant le degré d'autonomie de ces touristes. Certains auteurs utilisent le terme backpackers pour désigner ces touristes qui constitue une cible privilégiée pour les éditeurs de guides de voyage. La faible importance des sites dédiés au ciné-tourisme chez les voyagistes invite à se pencher sur cette catégorie de voyageurs afin de savoir s'ils sont plus concernés par le tourisme induit par le cinéma. Pour cela, la source et principal outil de recherche, hormis l'enquête directe auprès des touristes eux-mêmes, demeure le guide de voyage. Les guides de voyage s'adressent en effet, en théorie, à des touristes qui ne passent pas par des vecteurs d'organisation matérielle faisant partie de l'industrie touristique (c.-à.-d. des voyagistes). La multiplicité des renseignements pratiques qu'ils contiennent autorise l'individu à organiser son voyage lui-même avec ce seul outil. 
On peut donc légitimement demander comment le cinétourisme est décrit dans les guides de voyage. Y est-il important ou aussi faiblement représenté que chez les voyagistes? Pour répondre à cette question, on utilisera ici les contenus francophone et anglophone des guides Lonely Planet du Maroc et de la Tunisie. Le choix de se focaliser sur cet éditeur en particulier est justifié par son lectorat très large, diversifié, et surtout par le caractère mondialisé de ces guides de voyages, dont la diffusion peut aisément être qualifiée de planétaire. En effet, les guides Lonely Planet touchent un grand nombre de touristes de toutes nationalités en raison de leur grande diffusion dans le monde entier, et ce, dans un nombre très important de langues.

Les éditions revendiquent quelques 500 titres en anglais, près de 6 millions de guides vendus chaque année, et Lonely Planet a atteint, en août 2006, le chiffre de 80 millions de guides imprimés depuis sa création.

Si on s'intéresse tout d'abord, dans le contenu des guides, aux sites «à ne pas manquer » au Maroc comme en Tunisie, ni Ouarzazate ni Tataouine n'en font expressément partie, pas plus que d'autres sites locaux liés au cinéma (pour le Maroc, le guide mentionne comme « incontournables » Fès, la région du Toubkal, les gorges du Todra, Marrakech et Rabat; pour la Tunisie il s'agit de Tunis, Carthage, Sidi Bou Saïd, Kairouan et le Cap Bon).

En revanche, le guide montre bien l'importance du cinétourisme pour les voyageurs individuels dans le cas de la Tunisie, puisqu'il mentionne en effet une proposition de circuits personnalisés uniquement consacrés à Star Wars (Ham, $2008: 18)$ : il s'agit, "pour les inconditionnels de Star Wars", de visiter Onk Djemel, Sidi Bouhlel (plus connu sous le nom de «Star Wars Canyon»), le fameux hôtel Sidi Driss, et bien sûr les Ksour Hadada et Ouled Soltane, ce dernier ayant servi de décor au quartier des esclaves de Tatouïne.

Dans le guide anglophone (2009) consacré au Maroc, en revanche, Ouarzazate n'est pas du tout considérée comme une ville de ciné-tourisme, contrairement au presque éloge fait à Star Wars dans la version française consacrée à la Tunisie. Ainsi, c'est presque comme une attraction pour enfants qu'est présentée la visite des Studios Atlas : «Movie buffs and their kids might get a kick at Atlas Films Corporation Studios» (Clammer, 2009 : 342). On se demande même si ce n'est pas l'ironie qui est de mise quelques lignes plus loin : "Who knows, you may even get discovered by a talent scout looking for extras!»

Le guide opère donc un traitement différencié entre les deux attractions ciné-touristiques que constituent le Sud tunisien et les Studios Atlas. D'un côté la visite des installations et décors ressemble plus à une visite folklorique et amusante, en raison de son supposé aspect factice et mondialisé (au sens négatif du terme); de l'autre, en dépit de sa relative marginalité dans les grands circuits tunisiens, la visite des sites associés à Star Wars revêt une certaine importance aux yeux des fanatiques et font l'objet d'une certaine sacralisation, dont Gagnon (2007: 8) rappelle l'importance pour MacCannell. Ce traitement différencié des deux lieux de ciné-tourisme est paradoxalement inversement proportionnel à leur développement touristique respectif. En effet, alors que le Sud tunisien n'a pas connu d'aménagements d'ampleur liés au cinéma et au tourisme (il suffit pour s'en convaincre de voir comment l'hôtel Ksar Hadada est rapidement tombé dans l'abandon, et de comparer l'hôtel Sidi Driss aux "palaces» de Ouarzazate), le Lonely Planet valorise fortement la visite des sites liés à Star Wars. Dans le même temps, le "Ouallywood» (Clammer, 2009 : 342) du Sud marocain est présenté comme une attraction à visiter rapidement, voire à ne pas visiter, et comme un site touristique assez banal, en comparaison avec les palmeraies, les casbahs ou le désert à proprement parler.

\section{Conclusion}

Si le désert est bien considéré comme une seule et même ressource cinématographique exploitable au sens large dans le Sahara tunisien et marocain, les conséquences de cette exploitation diffèrent fortement d'un cas à l'autre.

Ainsi, les paysages de Star Wars apparaissent, dans le cas de la Tunisie, comme un élément relativement confidentiel et préservé, peu proposé par les voyagistes, et mobilisés essentiellement par un public de passionnés à la recherche d'un espace imaginaire précis, la planète Tatouïne. La connaissance préalable de la saga cinématographique est nécessaire pour organiser la visite de sites, la plupart du temps organisée par des voyageurs individuels et autonomes, comme le montre l'existence d'un guide très confidentiel et non publié intitulé Tataouine Tours: On location guidebook, Travel guidebook for Star Wars filming locations in Tunisia. Le ciné-tourisme au Maroc, essentiellement basé aux alentours de Ouarzazate, diffère quant à lui de celui de la Tunisie, car il concerne des studios, c'est-à-dire des structures de production héritées des premiers tournages dans les années 1970. Dans le premier cas, il n'y a qu'une seule production cinématographique, pour plusieurs microsites éparpillés; dans le second cas, plusieurs productions, pour un site majeur à visiter, celui des Studios Atlas, dans le cadre d'un tourisme plus «massif».

Enfin, les deux cas étudiés ici présentent également un point commun, car le ciné-tourisme y apparaît surtout comme une pratique touristique assez pauvre en marqueurs, qu'ils soient on-sight ou off-sight. Il n'est donc pas à proprement parler une pratique touristique, du moins en tant qu'objectif, mais plutôt une activité périphérique incluse dans un séjour.

\section{Références}

ANONYME (2003) «Un mécène est mort», Maroc Hebdo International, 543 , p. 39.

BLEASDALE, Sue (2006) "Connecting Paradise, Culture and Tourism in Tunisia”, Journal of Intercultural Studies, vol. 27, nº 4, p. 447-460.

BOUAOUINATE, Asmae (2008) «Les acteurs locaux du tourisme de désert au Maroc : le cas de l'erg Chebbi et de Zagora-Mhamid». Thèse de doctorat de géographie, Rabat : IRD. 612 p.

BRAULT, Frédérick (2004) Le tourisme et la transformation du territoire et du paysage au Maroc, UNESCO. Papier présenté dans le cadre du Workshop de la CUPEUM. Marrakech. 25 novembre au 4 décembre.

BROOKER, Will (2002) Using the force. Creativity, community and Star Wars fans, Londres : Continuum. $230 \mathrm{p}$. 
BUSBY, Graham et Julia KLUG (2001) "Movie-induced tourism: The challenge of measurement and other issues", Journal of Vacation Marketing, $\mathrm{n}^{\circ}$ 7, p. 316-332.

CARLA, Daniela; Sara KINDOMB et Karen SMITH (2007) “Tourists Experiences of Film Locations: New Zealand as 'Middle-Earth', Tourism Geographies, vol. 9, n 1 , p. 49-63.

CAZES, Georges (2005) «Le désir, dimension trop occultée dans la recherche géographique. Considérations à partir du tourisme», Géographie et Cultures, $\mathrm{n}^{\circ}$ 53, p. 123-130.

CLAMMER, Paul (2009) Lonely Planet Morocco, Londres : Lonely Planet ed. $528 \mathrm{p}$.

COHEN, Erik (1972), “Towards a sociology of international tourism”, Social Research, vol. 27, $\mathrm{n}^{\circ}$ 1, p. 164-182.

DESAUBES, Bernadette (1997) «Quand Avoriaz faisait son cinéma», Revue Espaces, $\mathrm{n}^{\circ} 146$, p. 11-14.

FRANÇOIS, Guy-Claude (1999) «Au cinéma, le paysage est un acteur», chapitre 7 DANS MOTTET, Jean (sous la direction de), Les paysages $d u$ cinéma, p. 87-91. Seyssel : Champ Vallon.

FREYTAG, Tim (2008) "Making a difference: tourist practices of repeat visitors in the city of Paris", Social Geography Discussions, n 4, p. 1-25.

GAGNON, Serge (2007) «Attractivité touristique et «sens» géo-anthropologique des territoires", Téoros, vol. 26, nº 2, p. 5-11.

GOLDSMITH, Ben et Tom O'REGAN (2003) Cinema Cities, Media Cities : The Contemporary International Studio Complex, Brisbane : Australian Key Centre for Cultural and Media Policy. 118 p.

HAM, Anthony (2008) Lonely Planet Tunisie, Londres : Lonely Planet ed. $311 \mathrm{p}$.

MaCCANNELL, Dean (1989a) The tourist : a new theory of the leisure class, Los Angeles : University of California Press. 229 p.
MaCCANNELL, Dean (1989b), "Introduction : Semiotic of Tourism", Annals of Tourism Research, $\mathrm{n}^{\circ}$ 16, p. 1-6.

MINVIELLE, Jean-Paul; Mounir SMIDA et Wided MADJOUB (2007) Tourisme saharien et développement durable. Enjeux et approches comparatives, Sousse : Université de Sousse. 649 p.

MOTTET, Jean (1998) L'invention de la scène américaine: cinéma et paysage, Paris : L'Harmattan. $280 \mathrm{p}$.

MOTTET, Jean (1999) Les paysages du cinéma, Seyssel : Champ Vallon. $261 \mathrm{p}$.

ORUETA, Agustín et Carlos VALDES (2007) «Cine y geografia : espacio geografico, paisaje y territorio en las producciones cinematográficas», Boletín de la Asociación de Geógrafos Españoles, nº 45, p. 157-190.

PIEROZAK, Isabelle et Didier De ROBILLARD (2004) «Quand la langue fait son cinéma : l'univers linguistique et sociolinguistique de Star Wars 1 : la menace fantôme dans sa version française», dans ROSSIGENSANE, Nathalie (sous la direction de), Mélanges en l'honneur de Nicole Gueunier, Littérature et Nation, p. 335-355. Tours : Publications de l'Université François-Rabelais.

RIEUCAU, Jean et Jérôme LAGEISTE (2006) L'empreinte du tourisme. Contribution à l'identité du fait touristique, Paris : L'Harmattan. 342 p.

RILEY, Roger; Dwayne BAKER et Carlton S. VAN DOREN (1998) "Movieinduced tourism”, Annals of Tourism Research, vol. 25, nº 4, p. 919-935.

RILEY, Roger et Carlton S. VAN DOREN (1992) "Movies as tourism promotion: A 'pull' factor in a 'push' location", Tourism management, vol. 13, n 3, p. 267-274.

TUAN, Yi-Fu (1974) Topohilia : study of environmental perception, attitudes and values, Englewood Cliffs : Prentice Hall. 260 p.

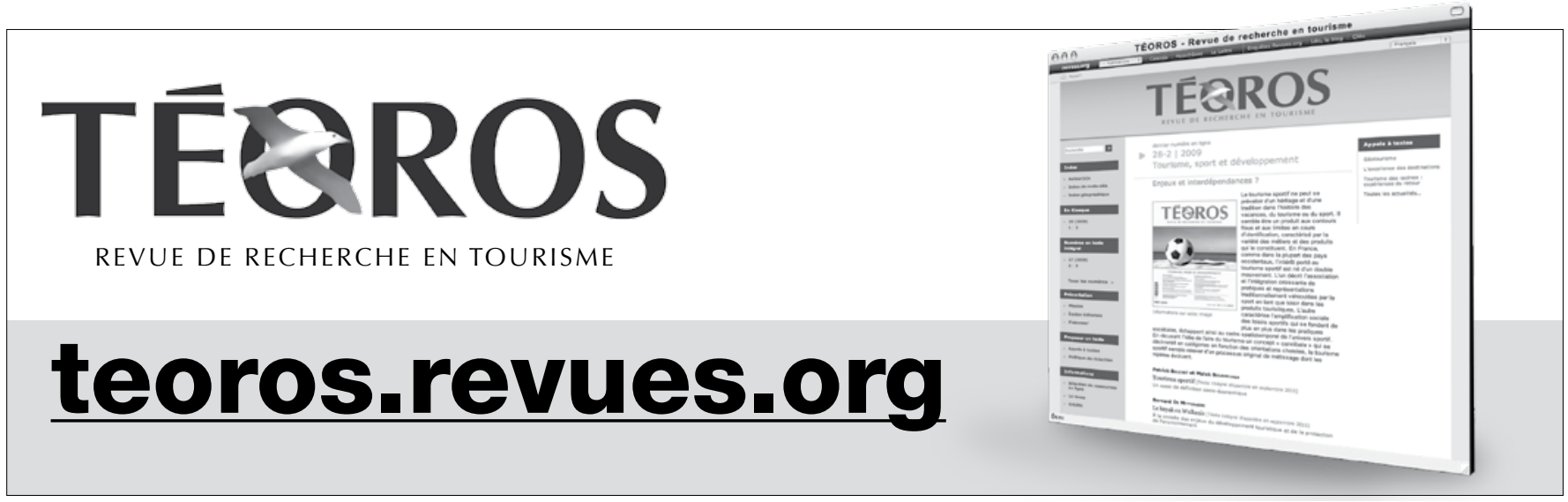

TÉOROS, vol. 30, n 1, p. 90-98 @ 2011 